Pacific

Journal of

Mathematics

CONNECTIONS ON PRINCIPAL BUNDLES OVER CURVES IN POSITIVE CHARACTERISTICS

INDRANIL Biswas and S. SUbramanian 


\title{
CONNECTIONS ON PRINCIPAL BUNDLES OVER CURVES IN POSITIVE CHARACTERISTICS
}

\author{
INDRANIL Biswas AND S. Subramanian
}

Let $X$ be an irreducible smooth projective curve over an algebraically closed field $k$ of characteristic $p$, with $p>5$. Let $G$ be a connected reductive algebraic group over $k$. Let $H$ be a Levi factor of some parabolic subgroup of $G$ and $\chi$ a character of $H$. Given a reduction $E_{H}$ of the structure group of a $G$-bundle $E_{G}$ to $H$, let $E_{\chi}$ be the line bundle over $X$ associated to $E_{H}$ for the character $\chi$. If $G$ does not contain any $\mathrm{SL}(n) / Z$ as a simple factor, where $Z$ is a subgroup of the center of $\operatorname{SL}(n)$, we prove that a $G$-bundle $E_{G}$ over $X$ admits a connection if and only if for every such triple $\left(H, \chi, E_{H}\right)$, the degree of the line bundle $E_{\chi}$ is a multiple of $p$. If $G$ has a factor of the form $\mathrm{SL}(n) / Z$, then this result is valid if $n$ is not a multiple of $p$. If $G$ is a classical group but not of the form $\operatorname{SL}(n) / Z$, then this criterion for the existence of connection remains valid even if $p \geq 3$.

\section{Introduction.}

Let $X$ be an irreducible smooth projective curve over an algebraically closed field $k$. Take a vector bundle $E$ over $X$. A subbundle $V$ of $E$ is called a direct summand if the quotient homomorphism $E \longrightarrow E / V$ splits. For $k=\mathbb{C}$, a theorem of Andre Weil says that $E$ admits a connection if and only if every direct summand of $E$ is of degree zero [10].

Let $G$ be a connected reductive algebraic group over $k$. Let $E_{G}$ be a principal $G$-bundle over $X$. Our aim is to give a criterion for the existence of a connection on $E_{G}$. Note that since the dimension of $X$ is one, the curvature of a connection on $E_{G}$ must vanish. In other words, any connection on $E_{G}$ is automatically flat.

Let $H \subseteq G$ be a Levi factor of a parabolic subgroup of $G$. Let $E_{H} \subseteq E_{G}$ be a reduction of structure group of $E_{G}$ to $H$. Take a character

$$
\chi: H \longrightarrow k^{*}
$$

of $H$ and consider the line bundle $E_{\chi}:=\left(E_{H} \times k\right) / H$ associated to $E_{H}$ for the character $\chi$. A connection on $E_{G}$ induces a connection on $E_{H}$ (Proposition 2.2), which, in turn, induces a connection on the line bundle $E_{\chi}$. 
It is well-known that a line bundle $\xi$ over $X$ admits a connection if and only if the degree of $\xi$ is a multiple of the characteristic of $k$ (see also Corollary 2.1). Therefore, if $E_{G}$ admits a connection then the degree of any line bundle $E_{\chi}$ of the above type must be a multiple of the characteristic of $k$.

Let $p$ denote the characteristic of $k$. We will assume that $p>5$.

Let $Z(\mathrm{SL}(n)) \subset \mathrm{SL}(n)$ be the center. First assume that $G$ does not have a simple factor of the form $\operatorname{SL}(n) / Z$, where $Z \subseteq Z(\mathrm{SL}(n))$. We prove that if the degree of any $E_{\chi}$ of the above type is a multiple of $p$, then $E_{G}$ admits a connection (Theorem 2.3).

If $G$ contains a simple factor of the form $\operatorname{SL}(n) / Z$, where $Z \subseteq Z(\operatorname{SL}(n))$, and $p$ does not divide $n$, then $E_{G}$ admits a connection if and only if the degree of any line bundle $E_{\chi}$ of the above type is a multiple of $p$.

If $G$ is a classical group but not $\mathrm{SL}(n) / Z$, where $Z \subseteq Z(\mathrm{SL}(n))$, then the condition $p>5$ can be relaxed to $p>2$.

\section{The Atiyah bundle.}

Let $H$ be an algebraic group over $k$. Take a principal $H$-bundle $E_{H}$ over $X$. The projection of the total space of $E_{H}$ to $X$ will be denoted by $\psi$. For any open subset $U$ of $X$, consider the space of $H$-invariant vector fields on $\psi^{-1}(U)$ for the natural action of $H$ on the fibers of $\psi$. This gives rise to a vector bundle $\operatorname{At}\left(E_{H}\right)$ on $X$ known as the Atiyah bundle.

Let $\mathfrak{h}$ denote the Lie algebra of $H$. Consider the adjoint action of $H$ on $\mathfrak{h}$. The associated vector bundle $\left(E_{H} \times \mathfrak{h}\right) / H$, known as the adjoint bundle, will be denoted by $\operatorname{ad}\left(E_{H}\right)$. Note that $\operatorname{ad}\left(E_{H}\right)$ corresponds to the sheaf of $H$-invariant vertical vector fields on $E_{H}$. Therefore, we have an exact sequence

$$
0 \longrightarrow \operatorname{ad}\left(E_{H}\right) \longrightarrow \operatorname{At}\left(E_{H}\right) \longrightarrow T X \longrightarrow 0
$$

of vector bundles over $X$. This sequence is known as the Atiyah exact sequence.

A connection on $E_{H}$ is a splitting of the exact sequence (2.1) [1], [9]. See Section 5 of [7] for connections on vector bundles in positive characteristics.

Note that both the sheaves $\operatorname{At}\left(E_{H}\right)$ and $T X$ are equipped with a Lie algebra structure induced by the Lie bracket operation of vector fields. Given a splitting

$$
\sigma: T X \longrightarrow \operatorname{At}\left(E_{H}\right)
$$

of the Atiyah exact sequence, consider the homomorphism

$$
\bar{\sigma}: T X \otimes T X \longrightarrow \operatorname{ad}\left(E_{H}\right)
$$

defined by $s \otimes t \longmapsto[\sigma(s), \sigma(t)]-\sigma([s, t])$, where $s$ and $t$ are local sections of $T X$, which is known as the curvature. Since $p \neq 2, \bar{\sigma}$ is skew-symmetric and $\operatorname{dim} X=1$, we have $\bar{\sigma}=0$. In other words, any connection on $X$ is flat. 
Set $H=\mathrm{GL}(n)$. So, using the standard representation of $\mathrm{GL}(n), E_{H}$ corresponds to a rank $n$ vector bundle $V$ over $X$. The exact sequence (2.1) becomes

$$
0 \longrightarrow \operatorname{End}(V) \longrightarrow \operatorname{At}(V) \longrightarrow T X \longrightarrow 0,
$$

where $\operatorname{At}(V)$ is the subbundle of the sheaf of differential operators $\operatorname{Diff}_{X}^{1}(V, V)$ defined by the condition that the image by the symbol homomorphism $\operatorname{Diff}_{X}^{1}(V, V) \longrightarrow T X \otimes \operatorname{End}(V)$ is contained in the subbundle $T X \otimes \operatorname{Id}_{V}$.

Consider the extension class

$$
\tau \in H^{1}\left(X, K_{X} \otimes \operatorname{End}(V)\right)
$$

for the exact sequence (2.2), where $K_{X}$ is the canonical bundle of $X$. Using the trace homomorphism $\operatorname{tr}: \operatorname{End}(V) \longrightarrow \mathcal{O}_{X}$, we have

$$
\operatorname{tr}(\tau) \in H^{1}\left(X, K_{X}\right)=k
$$

( $k$ is the base field), where the identification $H^{1}\left(X, K_{X}\right)=k$ is the one given by Serre duality.

Let $d \in \mathbb{Z}$ be the degree $V$, which is same as the degree of the line bundle $\bigwedge^{n} V$. The image of $d$ in $k$ by the obvious homomorphism $\mathbb{Z} \longrightarrow k$ coincides with $\operatorname{tr}(\tau)$.

Consequently, if a $\mathrm{GL}(n)$-bundle admits a connection, then the degree of the corresponding rank $n$ vector bundle is a multiple of $p$, the characteristic of $k$.

This observation and the above identity $d=\operatorname{tr}(\tau)$ together have the following corollary:

Corollary 2.1. A line bundle $\xi$ over $X$ admits a connection if and only if the degree of $\xi$ is a multiple of $p$ (possibly zero).

The above corollary is well-known [7, p. 190, Theorem 5.1] (in [7] this Theorem 5.1 is attributed to P. Cartier), [8].

As in the introduction, let $G$ be a connected reductive algebraic group over $k$. Let $P$ be a parabolic subgroup of $G$. Let $R_{u}(P)$ denote the unipotent radical of $P$. The quotient group $P / R_{u}(P)$ is called the Levi factor of $P$ [4]. The projection $P \longrightarrow P / R_{u}(P)$ splits in the sense that there is a connected closed reductive subgroup $H$ of $P$ which projects isomorphically to $P / R_{u}(P)$. However, there may be more than one such subgroup. We will call a subgroup $H$ of $P$ with this property a Levi factor of $P$.

Take a $G$-bundle $E_{G}$ over $X$. Suppose

$$
\sigma: X \longrightarrow E_{G} / H
$$

be a reduction of structure group of $E_{G}$ to a Levi factor $H$. So, the inverse image $q^{-1}(\sigma(X))$, where $q: E_{G} \longrightarrow E_{G} / H$ is the obvious quotient map, is an $H$-bundle. This $H$-bundle will be denoted by $E_{H}$. 
Fix a character $\chi: H \longrightarrow k^{*}$ of $H$. Consider the quotient

$$
E_{\chi}:=\left(E_{H} \times k\right) / H
$$

for the diagonal action of $H$, where $H$ acts on $k$ through $\chi$, which is a line bundle over $X$. We recall that the diagonal action of any $g \in H$ sends a point $(z, t) \in E_{H} \times k$ to $\left(z g, \chi\left(g^{-1}\right) t\right)$.

Proposition 2.2. If $E_{G}$ admits a connection, then the degree of the line bundle $E_{\chi}$ is a multiple of $p$.

Proof. Any connection on $E_{H}$ induces a connection on $E_{\chi}$. Therefore, in view of Corollary 2.1 it suffices to show that any connection on $E_{G}$ induces a connection on $E_{H}$.

Let $\mathfrak{g}$ (respectively, $\mathfrak{h}$ ) denote the Lie algebra of $G$ (respectively, $H$ ). Since $H$ is a Levi factor, there exists a $H$-equivariant splitting

$$
f: \mathfrak{g} \longrightarrow \mathfrak{h}
$$

of the inclusion homomorphism of $\mathfrak{h}$ in $\mathfrak{g}$. Indeed, if $\mathfrak{p}$ and $\mathfrak{q}$ are two opposite parabolics containing $\mathfrak{h}$ as the common Levi factor, then the direct sum of the radicals of $\mathfrak{p}$ and $\mathfrak{q}$ is a $H$-invariant complement of $\mathfrak{h}$.

We recall that a connection on $E_{G}$ is a $\mathfrak{g}$-valued 1-form $\omega$ on $E_{G}$ satisfying the two conditions:

1) For any $v \in \mathfrak{g}$, the evaluation of $\omega$ on the vector field corresponding to $v$ coincides with the constant function $v$;

2) the form $\omega$ is equivariant for the action of $G$ on $E_{G}$ and the adjoint action of $G$ on its Lie algebra $\mathfrak{g}$.

The kernel of such a form $\omega$ defines a splitting of the Atiyah exact sequence (2.1). To explain this, let $\psi$ denote the projection of $E_{G}$ to $X$. Given a tangent vector $v \in T_{x} X$, where $x \in X$, and a point $z \in \psi^{-1}(x) \subset E_{G}$, there is a unique tangent vector $w \in T_{z} E_{G}$ projecting to $v$ that is contained in the kernel of the form $\omega$. This way we get a section $v^{\prime}$ of $T E_{G}$ over $\psi^{-1}(x)$. This section $v^{\prime}$ is clearly $G$-invariant. In other words, $v^{\prime}$ gives $n$ element $v^{\prime \prime}$ of the fiber $\operatorname{At}\left(E_{G}\right)_{x}$. Sending any $v$ to $v^{\prime \prime}$ we obtain a splitting of the Atiyah exact sequence (2.1). Conversely, given a splitting $\sigma: T X \longrightarrow \operatorname{At}\left(E_{G}\right)$ of the Atiyah exact sequence, it is easy to see that there is a unique one-form $\omega$ satisfying the above two conditions such that the kernel of $\omega$ is the image of $\sigma$.

Given a connection on $E_{G}$ defined by a one-form $\omega$, let $\omega^{\prime}$ denote the restriction of $\omega$ to $E_{H} \subseteq E_{G}$. Now consider the $\mathfrak{h}$-valued one-form

$$
\bar{\omega}:=f \circ \omega^{\prime}
$$

on $E_{H}$, where $f$ is defined in $(2.3)$.

It is easy to check that the form $\bar{\omega}$ satisfies the two conditions needed to define a connection on $E_{H}$. Consequently, existence of a connection on $E_{G}$ 
ensures the existence of a connection on $E_{H}$. This completes the proof of the proposition.

As before, let $Z(\mathrm{SL}(n))$ denote the center of $\mathrm{SL}(n)$.

Following is the main result proved here:

Theorem 2.3. Let $p>5$ and assume that $G$ does not contain $\operatorname{SL}(n) / Z$ as a simple factor, where $Z \subseteq Z(\mathrm{SL}(n))$. $A$ G-bundle $E_{G}$ over $X$ admits a connection if and only if for every pair $(H, \chi)$, where $\chi$ is a character of the Levi factor $H$ of some parabolic subgroup, the degree of the line bundle $E_{\chi}$ is a multiple of $p$. If there is a subgroup $Z \subseteq Z(\mathrm{SL}(n))$ such that $G$ contains $\mathrm{SL}(n) / Z$ as a simple factor, then same criterion is valid if $p>5$ and $p$ does not divide $n$.

Since $H$ is reductive, Proposition 2.2 says that if $E_{G}$ admits a connection, then the line bundle $E_{\chi}$ is a multiple of $p$. We will complete the proof of the theorem in Section 4. In the next section we will show that it suffices to prove for simple groups.

\section{Reduction to the case of simple groups.}

Let $Z(G) \subset G$ denote the reduced center of $G$. Let

$$
G^{\prime}:=G / Z(G)
$$

be the quotient. Consider the commutator $[G, G]$, and let

$$
Z=G /[G, G]
$$

be the quotient. So $G^{\prime}$ is a semisimple quotient of $G$ and $Z$ is an abelian quotient of $G$.

For a principal $G$-bundle $E_{G}$ on $X$, let $E_{G^{\prime}}$ (respectively, $E_{Z}$ ) denote the principal $G^{\prime}$-bundle (respectively, principal $Z$-bundle) obtained by extending the structure group of $E_{G}$ using the obvious projection of $G$ to $G^{\prime}$ (respectively, $Z$ ).

Lemma 3.1. The $G$-bundle $E_{G}$ admits a connection if and only if both $E_{G^{\prime}}$ and $E_{Z}$ admit connection.

Proof. Since $E_{G^{\prime}}$ and $E_{Z}$ are extensions of structure group of $E_{G}$, any connection on $E_{G}$ induces connection on $E_{G^{\prime}}$ and $E_{Z}$.

Note that the fiber product $E_{G^{\prime}} \times_{X} E_{Z}$ is a principal $\left(G^{\prime} \times Z\right)$-bundle. Let

$$
\rho: G \longrightarrow G^{\prime} \times Z
$$

be the diagonal homomorphism induced by the projections of $G$ to $G^{\prime}$ and $Z$. Since the kernel of $\rho$ is finite and it induces an isomorphism of Lie algebras, the natural map $E_{G} \longrightarrow E_{G^{\prime}} \times_{X} E_{Z}$ is an étale covering map. Consequently, the Atiyah exact sequence for $E_{G}$ and $E_{G^{\prime}} \times_{X} E_{Z}$ coincide. 
It is easy to see that if

$$
0 \longrightarrow \operatorname{ad}\left(E_{G^{\prime}}\right) \longrightarrow \mathcal{A} \stackrel{f_{1}}{\longrightarrow} T X \longrightarrow 0
$$

and

$$
0 \longrightarrow \operatorname{ad}\left(E_{Z}\right) \longrightarrow \mathcal{B} \stackrel{f_{2}}{\longrightarrow} T X \longrightarrow 0
$$

are the Atiyah exact sequences for $E_{G^{\prime}}$ and $E_{Z}$ respectively, and $p$ (respectively, $q$ ) is the obvious projection of $\mathcal{A} \oplus \mathcal{B}$ to $\mathcal{A}$ (respectively, $\mathcal{B}$ ), then the exact sequence

$$
0 \longrightarrow \operatorname{ad}\left(E_{G^{\prime}}\right) \oplus \operatorname{ad}\left(E_{Z}\right) \longrightarrow \operatorname{kernel}\left(f_{1} \circ p-f_{2} \circ q\right) \subset \mathcal{A} \oplus \mathcal{B} \longrightarrow T X \longrightarrow 0
$$

obtained by combining the above two exact sequences is the Atiyah exact sequence for $E_{G^{\prime}} \times_{X} E_{Z}$. From this it follows that if the Atiyah exact sequences for $E_{G^{\prime}}$ and $E_{Z}$ split, then the Atiyah exact sequence for $E_{G^{\prime}} \times_{X}$ $E_{Z}$ also splits. Indeed, if

$$
\sigma_{1}: T X \longrightarrow \mathcal{A}
$$

and $\sigma_{2}: T X \longrightarrow \mathcal{B}$ are splittings of Atiyah exact sequences for $E_{G^{\prime}}$ and $E_{Z}$ respectively, then the diagonal homomorphism

$$
\left(\sigma_{1}, \sigma_{2}\right): T X \longrightarrow \mathcal{A} \oplus \mathcal{B}
$$

is the splitting of the Atiyah exact sequence for $E_{G^{\prime}} \times_{X} E_{Z}$. Therefore, if both $E_{G^{\prime}}$ and $E_{Z}$ admit connections then the $\left(G^{\prime} \times Z\right)$-bundle $E_{G^{\prime}} \times_{X} E_{Z}$ also admits a connection. This completes the proof of the lemma.

The group $Z$ is a product of copies of $k^{*}$, and $Z$ has exactly one parabolic subgroup which is $Z$ itself. Therefore, Theorem 2.3 is valid for $Z$.

The image of a parabolic subgroup $P$ of $G$ by the projection $G \longrightarrow G^{\prime}$ is a parabolic subgroup of $G^{\prime}$. Moreover, all parabolic subgroups of $G^{\prime}$ arise this way. The image, in $G^{\prime}$, of a Levi factor $H \subset P$ is a Levi factor of the corresponding parabolic subgroup of $G^{\prime}$.

Consequently, to establish Theorem 2.3 for $G$, it suffices to prove it for the semisimple group $G^{\prime}$.

Any parabolic subgroup of $G_{1} \times G_{2}$, where $G_{1}$ and $G_{2}$ are semisimple, is of the form $P_{1} \times P_{2}$, where $P_{i}$ is a parabolic subgroup of $G_{i}$. Furthermore, from the Proof of Proposition 2.2 it follows immediately that if we have $G_{i^{-}}$ bundle $E_{G_{i}}, i=1,2$, over $X$, then both $E_{G_{1}}$ and $E_{G_{2}}$ admit a connection if and only if the principal $\left(G_{1} \times G_{2}\right)$-bundle $E_{G_{1}} \times{ }_{X} E_{G_{2}}$ admits a connection. Therefore, it suffices to prove Theorem 2.3 under the assumption that $G$ is simple.

If $H \subset G$ is a Levi factor of a parabolic subgroup of $G$, and if $H_{1} \subset H$ is a Levi factor of a parabolic subgroup of $H$, then $H_{1}$, as a subgroup of $G$, is a Levi factor of some parabolic subgroup of $G$. Since $H_{1}$ is a Levi factor of $G$, using reverse induction, we may reduce the structure group of $G$ to such a situation where it does not admit any further reduction to some 
Levi factor. A Levi factor $H$ of some parabolic subgroup of $G$ will be called nontrivial if $H$ is a proper subgroup of $G$. Theorem 2.3 follows from the following theorem:

Theorem 3.2. Let $p>5$ and $G$ simple. Assume that either of the following two is valid:

1) $G$ is not isomorphic to $\mathrm{SL}(n) / Z$ for some subgroup $Z$ of the center $Z(\mathrm{SL}(n))$ of $\mathrm{SL}(n)$

2) if $G$ is isomorphic to $\mathrm{SL}(n) / Z$, where $Z \subseteq Z(\mathrm{SL}(n))$, then $p$ does not divide $n$.

Let $E_{G}$ be a $G$-bundle over $X$ such that $E_{G}$ does not admit any reduction of structure group to any nontrivial Levi factor. Such a $G$-bundle $E_{G}$ admits a connection.

This theorem will be proved in the next section.

\section{Obstruction for connection.}

Let $G$ be a simple algebraic group over $k$. As in Theorem 3.2, assume that either of the following two is valid:

1) $G$ is not isomorphic to $\mathrm{SL}(n) / Z$ for some subgroup $Z$ of the center $Z(\mathrm{SL}(n))$ of $\mathrm{SL}(n)$;

2) if $G$ is isomorphic to $\operatorname{SL}(n) / Z$, where $Z \subseteq Z(\operatorname{SL}(n))$, then $p$ does not divide $n$.

This assumption ensures that the Lie algebra $\mathfrak{g}$ of $G$ is isomorphic to $\mathfrak{g}^{*}$ as a $G$-module. Indeed, from $[\mathbf{5}, 0.13]$ we know that $\mathfrak{g}$ is simple. Now, as $\mathfrak{g}$ and $\mathfrak{g}^{*}$ are simple modules of same highest-weight, they are isomorphic $[\mathbf{6}$, p. 200, Proposition 2.4(a)]. In other words, $\mathfrak{g}$ is self-dual.

Consequently, for $G$-bundle $E_{G}$ we have $\operatorname{ad}\left(E_{G}\right)=\operatorname{ad}\left(E_{G}\right)^{*}$. Now the Serre duality gives

$$
H^{1}\left(X, K_{X} \otimes \operatorname{ad}\left(E_{G}\right)\right)=H^{0}\left(X, \operatorname{ad}\left(E_{G}\right)\right)^{*} .
$$

Assume that $E_{G}$ satisfies the conditions in Theorem 3.2. Let

$$
\tau \in H^{1}\left(X, K_{X} \otimes \operatorname{ad}\left(E_{G}\right)\right)
$$

be the extension class for the Atiyah exact sequence for $E_{G}$. Let

$$
\theta \in H^{0}\left(X, \operatorname{ad}\left(E_{G}\right)\right)^{*}
$$

be the functional that corresponds to $\tau$ by the isomorphism (4.1). Theorem 3.2 will be proved by showing that the functional $\theta$ vanishes identically. For this we need to study the section of $\operatorname{ad}\left(E_{G}\right)$.

For any $x \in X$ the fiber of $\operatorname{ad}\left(E_{G}\right)$ over $x$ will be denoted by $\operatorname{ad}\left(E_{G}\right)_{x}$. Note that $\operatorname{ad}\left(E_{G}\right)_{x}$ is isomorphic, as a Lie algebra, with $\mathfrak{g}$. 
Lemma 4.1. Let $\phi$ be a section of $\operatorname{ad}\left(E_{G}\right)$ such that for some point $x \in X$, the evaluation $\phi(x)$ is a nilpotent element of the Lie algebra $\operatorname{ad}\left(E_{G}\right)_{x}$. Then we have $\theta(\phi)=0$.

Proof. We noted earlier that the assumption on $G$ (stated at the beginning of this section) ensures that $\mathfrak{g}$ is simple. Therefore, an element $v$ of the simple Lie algebra $\mathfrak{g}$ is nilpotent if $\operatorname{ad}(v)$ is nilpotent. If $f$ is a $G$-invariant function on $\mathfrak{g}$, then evaluating $f$ on $\phi$ we get a function on $X$. Note that an element $v$ of $\mathfrak{g}$ is nilpotent if and only if all $G$-invariant functions on $\mathfrak{g}$ vanishing at $0 \in \mathfrak{g}$ also vanishes at $v$. Since $X$ is connected and complete there are no nonconstant functions on $X$. Consequently, if $\phi$ is nilpotent over some point, this observation implies that $\phi(y)$ is a nilpotent element of $\operatorname{ad}\left(E_{G}\right)_{y}$ for every point $y \in X$.

Using $\phi$ we will construct a reduction of structure group of $E_{G}$ to a parabolic subgroup of $G$. For that we will first construct a parabolic subalgebra bundle of $\operatorname{ad}\left(E_{G}\right)$.

Take any point $y \in X$ such that $\phi(y) \neq 0$. Let $V_{y}$ be the line in $\operatorname{ad}\left(E_{G}\right)_{y}$ generated by $\phi(y)$. Let $\mathfrak{n}_{y}^{1} \subset \operatorname{ad}\left(E_{G}\right)_{y}$ be the normalizer of $V_{y}$ and $\mathfrak{r}_{y}^{1} \subset \mathfrak{n}_{y}^{1}$ be the nilpotent radical.

Now inductively define $\mathfrak{n}_{y}^{i+1}$ to be the normalizer of $\mathfrak{r}_{y}^{i}$ in $\operatorname{ad}\left(E_{G}\right)_{y}$ and $\mathfrak{r}_{y}^{i+1}$ to be the nilpotent radical of $\mathfrak{n}_{y}^{i+1}$.

Let $\mathfrak{n}_{y}:=\lim \mathfrak{n}_{y}^{i}$ and $\mathfrak{r}_{y}:=\lim \mathfrak{r}_{y}^{i}$ be the limits of these two increasing sequences. From the construction of the two sequences it is obvious that $\mathfrak{n}_{y}$ is the normalizer, in $\operatorname{ad}\left(E_{G}\right)_{y}$, of $\mathfrak{r}_{y}$. Also, $\mathfrak{r}_{y}$ is the nilpotent radical of $\mathfrak{n}_{y}$. Therefore, $\mathfrak{n}_{y}$ is a parabolic subalgebra of $\operatorname{ad}\left(E_{G}\right)_{y}$. See $[4,30.3]$ for the details of this construction.

Consider the action of $G$ on itself by inner conjugation. Let $\operatorname{Ad}\left(E_{G}\right):=$ $\left(E_{G} \times G\right) / G$ be the gauge bundle (adjoint bundle) constructed using this action. Let $P_{y} \subset \operatorname{Ad}\left(E_{G}\right)_{y}$ be the parabolic subgroup of the fiber $\operatorname{Ad}\left(E_{G}\right)_{y}$ whose Lie algebra coincides with the parabolic subalgebra $\mathfrak{n}_{y}$ constructed above.

Since there are only finitely many conjugacy classes of parabolic subalgebras of $G$, there is a nonempty Zariski open subset $U$ of $X$ such that the conjugacy class of $\mathfrak{n}_{z}$ is independent of $z \in U$. Fix a parabolic subgroup $P$ of $G$ whose Lie algebra is in the same conjugacy class as $\mathfrak{n}_{z}$, where $z \in U$. To explain this with more details, we observe that the variety of nilpotent elements in $\mathfrak{g}$ is irreducible. Indeed, it is the image of $\mathfrak{g}$ by the Jordan decomposition. The variety of nilpotent elements in $\mathfrak{g}$ is filtered by conjugacy classes. Therefore, on some nonempty Zariski open subset of $X$, the evaluation of $\phi$ must lie in some particular stratum of this filtered variety.

Consider the obvious projection

$$
q(y):\left(E_{G}\right)_{y} \times G \longrightarrow \operatorname{Ad}\left(E_{G}\right)_{y} .
$$


Let $\left(E_{P}\right)_{y} \subset\left(E_{G}\right)_{y}$ be the subvariety consisting all elements $z$ such that $q(z, g) \in P_{z}$ for every $g \in P$. It is easy to check that $E_{P} \subset E_{G}$ is a reduction of structure group over $U$ of $E_{G}$ to the parabolic subgroup $P$ of $G$. Indeed, this is an immediate consequence of the fact that the normalizer of $P$ in $G$ is $P$ itself.

Since $G / P$ is a complete variety and $\operatorname{dim} X=1$, the reduction over $U$ extends to a reduction of structure group over $X$ of $E_{G}$ to $P$. Let $E_{P} \subset E_{G}$ denote this reduction of structure group.

Let $\operatorname{ad}\left(E_{P}\right) \subset \operatorname{ad}\left(E_{G}\right)$ be the adjoint bundle. The commutativity of the diagram

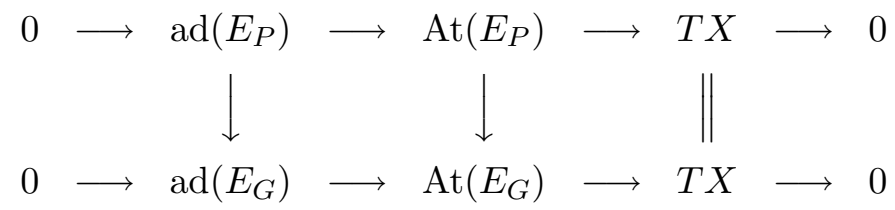

ensures that the cohomology class $\tau \in H^{1}\left(X, K_{X} \otimes \operatorname{ad}\left(E_{G}\right)\right)$ defined in (4.2) lies inside the image of $H^{1}\left(X, K_{X} \otimes \operatorname{ad}\left(E_{P}\right)\right)$ for the homomorphism defined by the inclusion of $\operatorname{ad}\left(E_{P}\right)$ in $\operatorname{ad}\left(E_{G}\right)$.

Now, since $\phi(y)$ is in the nilpotent radical $\mathfrak{r}_{y}$ of the parabolic subalgebra $\mathfrak{n}_{y}$, it follows that

$$
\theta(\phi)=0,
$$

where $\theta$ is defined in (4.3). Indeed, the subalgebra $\mathfrak{r}_{y}$ is contained in the annihilator of $\mathfrak{n}_{y}$ for each $y \in X$. Now, $\tau$ defined in (4.2) is in the image of $H^{1}\left(X, K_{X} \otimes \operatorname{ad}\left(E_{P}\right)\right)$. This immediately implies that for the nondegenerate pairing

$$
H^{1}\left(X, K_{X} \otimes \operatorname{ad}\left(E_{P}\right)\right) \otimes H^{0}\left(X, \operatorname{ad}\left(E_{P}\right)\right) \longrightarrow k
$$

defining the Serre duality, we have $\tau \otimes \phi \longmapsto 0$. In other words, $\theta(\phi)=0$. This completes the proof of the lemma.

In view of Lemma 4.1, to complete the proof of Theorem 3.2 it suffices to show that $\theta(\phi)=0$, where $\phi$ is a everywhere semisimple section of $\operatorname{ad}\left(E_{G}\right)$. Indeed, by the Jordan decomposition theorem, any section $\phi$ of $\operatorname{ad}\left(E_{G}\right)$ decomposes uniquely as $\phi_{s}+\phi_{n}$, where $\phi_{s}$ is everywhere semisimple and $\phi_{n}$ is everywhere nilpotent. So, to prove that the $\theta(\phi)=0$, it is enough to show that $\theta\left(\phi_{s}\right)=0$ and $\theta\left(\phi_{n}\right)=0$.

We will show that $\operatorname{ad}\left(E_{G}\right)$ does not admit any nonzero section which is semisimple everywhere. This is the content of the following lemma:

Lemma 4.2. Let $E_{G}$ be as in Theorem 3.2. Let $\phi \in H^{0}\left(X \operatorname{ad}\left(E_{G}\right)\right)$ be such that $\phi(y)$ is a semisimple vector of $\operatorname{ad}\left(E_{G}\right)_{y}$ for every $y \in X$. Then the section $\phi$ vanishes identically. 
Proof. Let $\phi$ be a nonzero section of $\operatorname{ad}\left(E_{G}\right)$ which is semisimple everywhere. Since $X$ is connected and complete, the characteristic polynomial for the adjoint action of $\phi(y)$ on $\operatorname{ad}\left(E_{G}\right)_{y}$ is independent of $y$. So $\phi$ does not vanish at point of $X$.

We have a decomposition

$$
\operatorname{ad}\left(E_{G}\right)_{y}=\bigoplus_{\lambda \in \Lambda} V_{y}^{\lambda}
$$

where $V^{\lambda}$ is the eigenspace for the eigenvalue $\lambda$ for the adjoint action of $\phi(y)$ on $\operatorname{ad}\left(E_{G}\right)_{y}$. So, $V_{y}^{0}$ coincides with the subalgebra of $\operatorname{ad}\left(E_{G}\right)_{y}$ that centralizes $\phi(y)$.

Let $\mathcal{V}_{y} \subset \operatorname{ad}\left(E_{G}\right)_{y}$ denote the direct sum of all eigenspaces in (4.2) with eigenvalue less than or equal to zero in the lexicographic ordering. Since

$$
\left[V_{y}^{\lambda_{1}}, V_{y}^{\lambda_{2}}\right] \subset V_{y}^{\lambda_{1}+\lambda_{2}}
$$

unless $\left[V_{y}^{\lambda_{1}}, V_{y}^{\lambda_{2}}\right]=0$, we have $\mathcal{V}_{y}$ as a subalgebra of $\operatorname{ad}\left(E_{G}\right)_{y}$.

Note that the direct sum of all eigenspaces in (4.5) with eigenvalue strictly positive (in the lexicographic ordering) is a nilpotent subalgebra. In other words, $\mathcal{V}_{y}$ has a complement which is nilpotent. Using [2, p. 473, Corollary 4.10], [3, p. 747, Lemma 4], it now follows that $\mathcal{V}_{y}$ is a parabolic subalgebra of $\operatorname{ad}\left(E_{G}\right)_{y}$ and $V_{y}^{0}$ its Levi factor.

Let $P_{y} \subset \operatorname{Ad}\left(E_{G}\right)_{y}$ be the parabolic subgroup with $\mathcal{V}_{y}$ as its Lie algebra. Let $H_{y}$ be the Levi factor of $P_{y}$ whose Lie algebra is $V_{y}^{0}$.

Let $\mathcal{W}_{y}$ denote the direct sum of all eigenspaces in (4.5) with eigenvalue greater than or equal to zero. Just as before, $\mathcal{W}_{y}$ is a parabolic subalgebra of $\operatorname{ad}\left(E_{G}\right)_{y}$ with $V_{y}^{0}$ as its Levi factor.

Let $Q_{y} \subset \operatorname{Ad}\left(E_{G}\right)_{y}$ be the parabolic subgroup whose Lie algebra is the direct sum of all eigenspaces in (4.5) with nonnegative eigenvalues. For the same reason as for $P_{y}$, the subgroup $H_{y}$ is a Levi factor of $Q_{y}$. Clearly we have $P_{y} \cap Q_{y}=H_{y}$.

It is easy to see that the conjugacy classes of $P_{y}$ and $H_{y}$ are independent of $y$. Fix subgroups $P, Q$ and $H$ in $G$ such that some identification of $G$ with $\operatorname{Ad}\left(E_{G}\right)_{y}$ takes them to $P_{y}, Q_{y}$ and $H_{y}$ respectively. Note that $P, Q$ and $H$ have been fixed independent of $y$. So $P \cap Q=H$ and $H$ is a Levi factor for both $P$ and $Q$.

As in the Proof of Lemma 4.1, using $P_{y}$ we have a reduction of structure group $E_{P} \subset E_{G}$ to $P$. More precisely, let $\left(E_{P}\right)_{y} \subset\left(E_{G}\right)_{y}$ be the subvariety consists of all $z$ with such that $q(y)(z) \in P_{x}$ for every $g \in P$, where $q(y)$ is the projection in (4.4). Similarly, we have a reduction of structure group $E_{Q} \subset E_{G}$ of $E_{G}$ to $Q \subset G$. Let

$$
E_{H}:=E_{P} \cap E_{Q} \subset E_{G}
$$


be the intersection of the two subvarieties $E_{P}$ and $E_{Q}$ of $E_{G}$. Clearly $E_{H}$ defines a reduction of structure group of $E_{G}$ to $P \cap Q=H$.

Recall the assumption in Theorem 3.2 that $E_{G}$ does not admit any reduction to a nontrivial Levi. Therefore, we have $H=G$. This immediately implies that $\phi=0$. This completes the proof of the lemma.

Lemma 4.1 and Lemma 4.2 together complete the proof of Theorem 3.2. It was noted in Section 3 that Theorem 3.2 completes the proof of Theorem 2.3. Therefore, the proof of Theorem 2.3 is complete.

If $G$ is a classical group but not isomorphic to $\mathrm{SL}(n) / Z$ for some subgroup $Z$ of the center $Z(\mathrm{SL}(n))$ of $\operatorname{SL}(n)$, then $\mathfrak{g}=\mathfrak{g}^{*}$ as a $G$-module if $p>2[\mathbf{5}$, 0.13]. Therefore, Theorem 2.3 remains valid in this case.

We note that for $G=E_{6}$, the $G$-module $\mathfrak{g}$ fails to be isomorphic to $\mathfrak{g}^{*}$ if $p=3[\mathbf{5}, 0.13$, p. 9$]$. For $G=E_{7}$, the $G$-module $\mathfrak{g}$ fails to be isomorphic to $\mathfrak{g}^{*}$ if $p=2[\mathbf{5}, 0.13$, p. 9$]$. For classical groups of type $B_{r}$ and $C_{r}$, we have $\mathfrak{g} \neq \mathfrak{g}^{*}$ if $p=2[5,0.13]$.

Acknowledgments. We thank the referee for helpful comments that helped in improving the exposition.

\section{References}

[1] M.F. Atiyah, Complex analytic connections in fibre bundles, Trans. Amer. Math. Soc., 85 (1957), 181-207, MR 19,172c, Zbl 0078.16002.

[2] A. Borel and F. Hirzebruch, Characteristic classes and homogeneous spaces, I, Amer. Jour. Math., 80 (1958), 458-538, MR 21 \#1586, Zbl 0097.36401.

[3] Harish-Chandra, Representations of semisimple Lie groups IV, Amer. Jour. Math., 77 (1955), 743-777, MR 17,282c, Zbl 0066.35603.

[4] J.E. Humphreys, Linear Algebraic Groups, Graduate Texts in Mathematics, 21, Springer-Verlag, New York, Heidelberg, Berlin, 1987, MR 53 \#633, Zbl 0471.20029.

[5] _ Conjugacy Classes in Semisimple Algebraic Groups, Mathematical Surveys and Monographs, 43, Amer. Math. Soc., 1995, MR 97i:20057, Zbl 0834.20048.

[6] J.C. Jantzen, Representations of Algebraic Groups, Pure and Applied Mathematics, 131, Academic Press, Boston, 1987, MR 89c:20001, Zbl 0654.20039.

[7] N.M. Katz, Nilpotent connections and the monodromy theorem: Applications of a result of Turrittin, Inst. Hautes Études Sci. Pub. Math., 39 (1970), 175-232, MR 45 \#271, Zbl 0221.14007.

[8] Algebraic solutions of differential equations p-curvature and the Hodge filtration, Invent. Math., 18 (1972), 1-118, MR 49 \#2728, Zbl 0278.14004.

[9] J.-L. Koszul, Lectures on Fibre Bundles and Differential Geometry, Reprint of the 1965 edition, T.I.F.R. Lectures on Mathematics and Physics, Springer-Verlag, Berlin, 1986, MR 88e:53040, Zbl 0607.53001.

[10] A. Weil, Généralisation des fonctions abéliennes, Jour. Math. Pures Appl., 17 (1938), 47-87, Zbl 0018.06302.

Received April 25, 2001 and revised April 17, 2002. 
School of Mathematics

Tata Institute of Fundamental Research

HOMI BHABHA ROAD

BOMBAY 400005

INDIA

E-mail address: indranil@math.tifr.res.in

School of Mathematics

Tata Institute of Fundamental Research

HOMI BHABHA ROAD

BOMBAY 400005

INDIA

E-mail address: subramnn@math.tifr.res.in 